\title{
Spectral Properties of One-Dimensional Schrödinger Operators with Potentials Generated by Substitutions
}

\author{
Anton Bovier ${ }^{1}$, Jean-Michel Ghez ${ }^{2,3}$ \\ ${ }^{1}$ Institut für Angewandte Analysis und Stochastik, Mohrenstrasse 39, D-10117 Berlin, Germany, \\ e-mail: bovier@iaas-berlin.d400.de \\ ${ }^{2}$ Centre de Physique Théorique, CNRS, Luminy, Case 907, F-13288 Marseille Cedex 9, France \\ ${ }^{3}$ Phymat, Département de Mathématiques, Université de Toulon et du Var, B.P. 132, F-83957 La \\ Garde Cedex, France-e-mail: ghez@cpt.univ-mrs.fr
}

Commun. Math. Phys. 158, 45-66 (1993)

Several people, and in particular Bert Hof, have pointed out to us that the definition of the potential on the negative integers given in our paper, as well as in some previous ones $[1,2]$ is actually inappropriate for several reasons and should be altered. The correct definition, which was for instance used in [3] in the case of the Fibonacci sequence, is to define the doubly infinite sequence $w$ as a limit point (in the product topology) of the periodic sequences $w^{(n)}$ that are obtained by repeating the words $\xi^{n}(0)$ indefinitely to the left and to the right, i.e.

$$
w^{(n)}=\ldots \xi^{n}(0) \xi^{n}(0) \xi^{n}(0) \xi^{n}(0) \ldots
$$

It is easy to see that $w \in \mathscr{A}^{Z}$ is then a fixpoint of some iterate of $\xi$, i.e. it is a fixpoint of some primitive substitution. The potential $V_{n}$ is defined by $V_{n}=v\left(w_{n}\right)$.

In all the proofs in our paper, as well as in $[1,2]$ this definition was implicitly used so that their results apply to such potentials rather than to those obtained by symmetric extensions. The crucial point where the former definition goes wrong is Lemma 3.1. Also, the unique ergodicity of the dynamical system $(\Omega, T)$ holds if $\Omega$ is the closure of the set of translates of $w$, but can fail if the old definition is adopted. The reference to the symmetry of the potential made in the proof of Lemma 3.4 is unnecessary. In fact, on p. 60 , the phrase "Assume that $\Psi_{E}(1) \neq 0$ (otherwise . . .)" should be removed. Note that the vector $\Psi_{E}(1)$ cannot be zero unless $\psi$ is identically zero.

Note also that there is a misprint on p. 60 . The last expression in the statement of Lemma 3.4 should be read $\left|x_{E}^{\left(n_{i}\right)}\left(\gamma_{0}\right)\right|$ instead of $\left|x_{E}^{\left(n_{i}\right)}(\beta)\right|$.

Finally, we would like to point out that the assumptions of Theorem 3 can be relaxed so that it applies to a larger class of substitution sequences, that comprises in particular the period-doubling sequence. This generalization has been suggested by L. Raymond [4] and goes as follows.

Note that $\mathscr{A}^{\star}$ has the structure of a free semi-group. We can easily extend this to a the free group $\hat{\mathscr{A}}^{\star}$ by adding the formal inverses of the letters in $\mathscr{A}$ as generators. $\xi$ has a natural extension as a group automorphism on $\hat{\mathscr{A}}^{\star}$. Moreover, the map 
$T_{E}$ provides a representation of this group if we set $T_{E}\left(\alpha^{-1}\right) \equiv\left[T_{E}(\alpha)\right]^{-1}$. With these notions in mind we can replace Theorem 3 by

Theorem 3. Suppose the hypothesis of Theorem 1 are satisfied. If in addition there exist $n_{0}, m<\infty$ such that $\xi^{n_{0}}(0)=\xi^{m}\left(\gamma_{0}\right) \Gamma \omega$, where

(i) $\gamma_{0} \in \mathscr{C}, \Gamma, \omega \in \hat{\mathscr{A}}^{\star}$,

(ii) $\Gamma=\xi^{m}\left(\gamma_{0}\right) \delta$, where $\delta \in \hat{\mathscr{A}}^{\star}$ has the property that there exists a finite integer $k$ such that $\xi^{k}(\delta)=\delta$,

then the spectrum of $H$ is purely singular continuous and supported on a Cantor set of zero Lebesgue measure.

The condition $\xi^{k}(\delta)=\delta$ implies that there is only a finite set of words $\xi^{n}(\delta)$, and as a consequence the norms of the matrices $\left[T_{E}\left(\xi^{n}(\delta)\right)\right]^{-1}$ are bounded uniformly in $n$. Using this fact, our proof applies with some obvious minor modifications under these hypothesis. Note that the previous assumptions cover the special case $\delta=i d$. Note that the period-doubling case is covered by these hypothesis with $n_{0}=2, m=1, \gamma_{0}=a, \delta=b^{-1} a, k=2$. This observation simplifies considerably the proof of the absence of eigenvalues in [1].

Acknowledgements. We would like to thank A. Hof, B. Host, and A. Sütő for having pointed out the problems arising from our previous definition of $V_{n}$, and $\mathrm{L}$. Raymond for suggesting the improvement in Theorem 3. Bert Hof has made valuable suggestions that help to improve the presentation of this erratum.

\section{References}

1. Bellissard, J., Bovier, A., Ghez, J.-M.: Spectral properties of a tight binding hamiltonian with period doubling potential. Commun. Math. Phys. 135, 379-399 (1991)

2. Bellissard, J., Bovier, A., Ghez, J.-M.: Gap labelling theorems for one dimensional discrete Schrödinger operators. Rev. Math. Phys. 4, 1-37 (1992)

3. Sütő, A.: The spectrum of a quasiperiodic Schrödinger operator. Commun. Math. Phys. 111, 409-415 (1987); Sütő, A.: Singular continuous spectrum on a Cantor set of zero Lebesgue measure for the Fibonacci hamiltonian, J. Stat. Phys. 56, 525-531 (1989)

4. L. Raymond, private communication

Communicated by $\mathrm{T}$. Spencer

Note added in proof: The above definition of $w$ is not general enough. One has to choose in $\mathscr{A}$ two letters $\alpha_{r}$ and $\alpha_{l}$, beginnings of a right (resp. left) fixpoint $u_{r}$ (resp $u_{l}$ ) of $\xi$ (or of some iterates of $\xi$ ) and such that the word $\alpha_{t} \alpha_{r}$ is contained in $u_{r}$. Then one defines $w$ by concatenation, that is $w=u_{l} u_{r}$. The previous definition of $w$ works only if one can choose $\alpha_{r}=\alpha_{l}=0$. We thank B. Hof, L. Raymond and A Sütő for this remark. 